\title{
UN REGISTRATORE DI INTELLIGIBILITÀ DEI SEGNALI RADIOTELEGRAFICI
}

\author{
Stelio Silleni
}

1. Introduzione. - Fra gli elementi di base per il progetto dei collegamenti radlio, sono particolarmente importanti e significativi: a) le caratleristiche della propagazione;

b) l'intensità di campo minima occorrente all'antenna ricevente, in presenza dei disturli.

Presso l'Istituto Nazionale di Geofisica vengono effettuate correntemente registrazioni di alcune caratteristiche della ionosfera (i) utili per yli elementi in a). Si è ora studiata la misura dell'intensità di campo minima occorrente all'antenna ricevente, in presenza dei disturbi, e per data intelligiloiliti.

Numerosi metodi sono stati studiati per questa misura (su cui in alcuni casi si riferisce con $\mathrm{i}$ concetti di "intensità dei disturbi » e "rapporto segnale'disturbo"). Su alcuni di questi studi abliamo riferito altrove $\bar{~} \%$. Tra questi metodi viene impiegato, per misure sistematiche effeltuate in diverse parti del mondo, un metodo di misura sintetica per confronto con un segnale, detto metorlo Thomas. Secondo il metodo Thomas ( ${ }^{1}$ ) (fig. 1 ) si ricevono i disturl,i con una antenna sequita da un preamplificatore a larga landa. Nel preamplificatore viene introdotto un segnale (Morse) a radiofrequenza, di intensita regolabile. Al preamplificatore segue un ricevitore. Un marconista riceve i segnali in cuffia, e regola l'intensita dell'oscillatore a radiofrequenza finché ottiene una intelligiloilitì del $95 \%$ dei caratteri. L'intensili del segnale impiegato viene allora registrata.

L'antenna, il preamplificatore, il ricevitore, la velocitì di trasmissione sono uguali per tutti i posti, allo scopo di ottenere risultati comparalibli. L'inconveniente principale del metodo è costituito dalla dipendenza da un elemento soggettivo (marconista), e purtroppo lo scarto nelle indicazioni che si ottiene con diversi marconisti è noterole ${ }^{1} \%$

$\mathrm{U}_{\mathrm{n}}$ metodo analogo è stato proposto $\left({ }^{7}\right)$ e usato $\left({ }^{6}\right)$ anche utilizzando mezzi automatici di ricezione (telescriventi). La applicazione 
dei risultati ad altri tipi di trasmissione crea però difficoltà anclıe concettuali; anche in questo caso nella misura interviene un elemento arbitrario - la telescrivente - ed una velocità di trasmissione obbligata.

Si è perciò studiata la possibilità di costruire un comparatore di segnali in cui non vi siano elementi arbitrari, e che sia sufficientemente versatile per permettere il confronto immediato fra le sue indicazioni di intellegribilità, e quelle rilevate da un marconista, da una telescri-

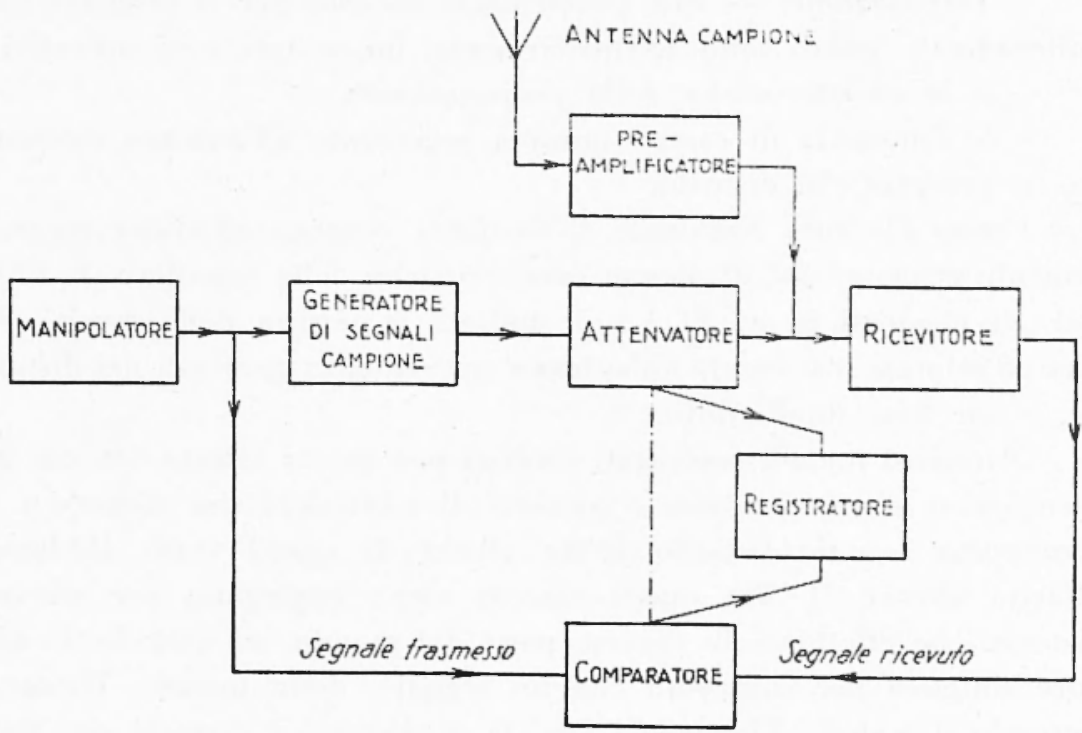

Fig. 1 - Metodo di misura sintetico ciei disturbi atmosferici, per confronto con un segnale cimpione. Nel metodo Thomas il comparatore $\dot{e} u n$ marconista.

vente, o da altri mezzi di ricezione. Un siffatto apparecchio può inoltre essere impiegato con un addestramento limitato. Si oppone alla automatizzazione completa la necessità di accertarsi, nel corso di una misura, che sul canale scelto non vi siano interferenze dovute ad altre emissioni. L'impiegro dell'apparecchio può, per converso, essere esteso anche alla misura della degradazione prodotta dalla presenza, sullo stesso canale o su canali adiacenti, di emissioni interferenti, ed al confronto fra diversi ricevitori e diverse antenne.

2. Definizioni. - Per chiarezza di comprensione si precisa il significato di alcune denominazioni impiegate nel seguito. 
Verranno considerati quei sistemi telegrafici che utilizzano due soli stati diversi. Questi stati verranno indicati come stato $O$ e stato 1 , e corrispondono, nei tipi di emissione radiotelegrafica più comuni, a:

$$
\begin{array}{lll}
\text { tipo di emissione } \quad \text { stato } \mathrm{O} & \text { stato } 1
\end{array}
$$

-radiotelegrafia A 1 . tasto alzato . tasto abbassato

(ad emissione " tutto . assenza di nota in . presenza di nota in o niente )) cuffia cuffia

- radiotelegrafia F 1 . emissione di una ra- .emissione di una ra(a spostamento di diofrequenza diofrequenza frequenza)

$$
\mathbf{f}-\Delta \mathbf{f} \quad \mathbf{f}+\Delta \mathbf{f}
$$

-posizione ( $\rightarrow$ ) di un -posizione $(+)$ di un relè polarizzato relè polarizzato

Segnale elementare o elemento ̀̀ uno dei due stati, mantenuto per un tempo $\tau$. Generalmente il tempo $\tau$ ̀̀ uguale per ciascun elemento, ed il suo valore è scelto in base alle caratteristiche del sistema telegrafico.

Messaggio è una successione di elementi in cui i due stati si seguono in modo determinato soltanto dalle proprietà statistiche del codice impiegato. La fiğ. 2 dà due esempi di messagrgi.
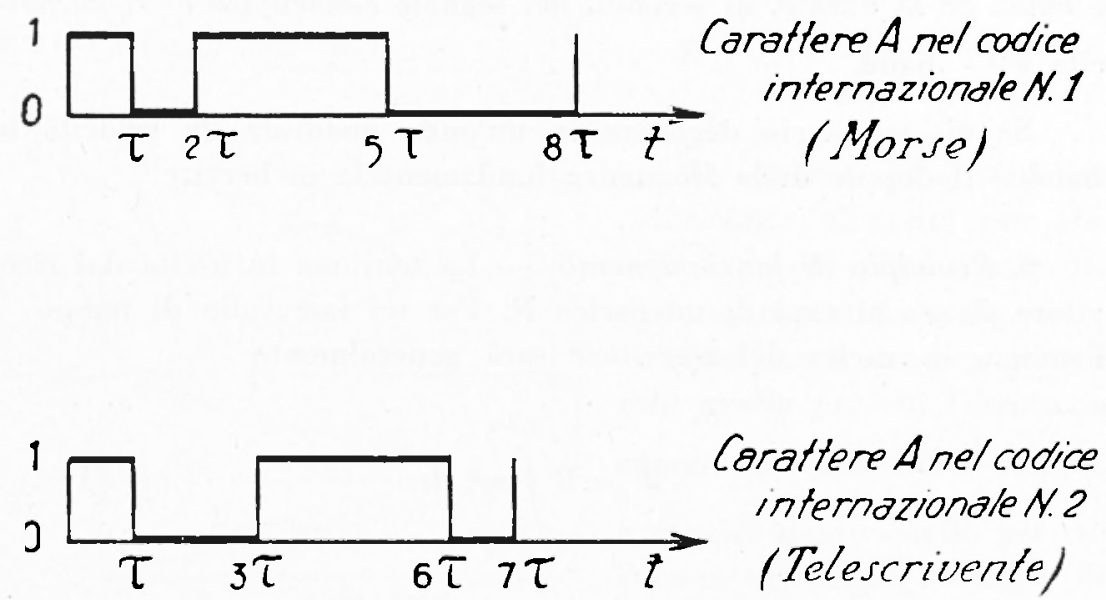

Fig. 2 - Esempi di messaggi in codice a 2 stati. 
Impulso, rettangolo, sono due forme d'onda (generalmente di tensionel rappresentate nella fig. $3 a, b$. Esse non sono necessariamente
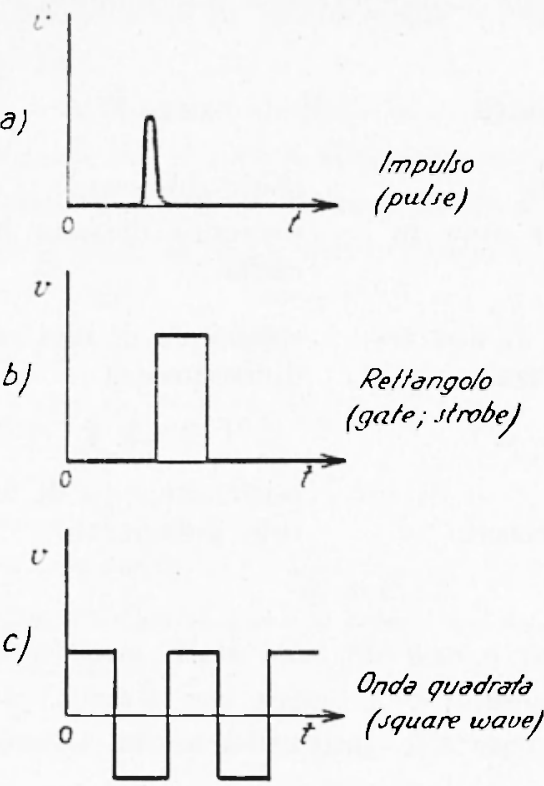

Fig. 3 - Denominazione di alcune forme d'onde. periodiche (questi due termini corrispondono ai termini ingrlesi pulse e gate o strobo).

Scatto è l'impulso che viene utilizzato per dare inizio ad un fenomeno, p. e. per sincronizzare un multiviloratore. (Corrisponde al termine inglese trigger).

Onda quadrata è una forma di tensione o corrente alternante simmetrica in cui il passagrgio da un valore estremo all'altro avviene in tempo trascurabile (rispetto ai tempi che interessano il fenomeno principale oggetto dello studiol. É rappresentata nella figr. $3 c$.

Velocità di trasmissione è l'inverso della durata del segnale elementare. Il nome dell'unità di misura della velocità di trasmissione è baud. Se la durata, in secondi, del segnale elementare è $T$, la velocità è $\frac{1}{\tau}$ baud.

Se un messaggio degenera in un'onda quadrata, la velocita in baud è il doppio della frequenza fondamentale in hertz.

3. Principio di funzionamento. - La tensione in uscita dal ricevitore sia $e_{\text {s, }}$ ai capi di un carico $R$. Per un intervallo di tempo $r$ l'energia in uscita del ricevitore sari generalmente

$$
W_{\mathrm{s}}=R \int_{i}^{t+\tau} e_{\mathrm{s}}^{2} d t
$$

Se la $\rho_{\mathrm{s}} \dot{e}$ in equilibrio statistico $\left(^{2}\right)$ la media fatta da un certo istante fino a $-\infty$ sarà uguale alla media fatta da $-\infty a+\infty$. 
Perciò si polrà assumere come espressione della energria media la

$$
W_{\ldots==} \frac{\tau R}{A} \lim _{A \rightarrow-\infty} \int_{-A}^{0} \mathrm{~s}^{2} d t
$$

Nel caso di ricezione in assenza di disturbi, il segnale elementare $O$ sarà rappresentato da un'energia $\Pi_{0}^{\prime}=0$, ed il segnale elementare 1 da un'energria $W_{1}^{\prime}=k W_{\mathrm{m}}^{\prime}$, in cui $k$ assume un valore dipendente dalla relativa frequenza stalistica dei due tipi di segnale clementare. Se la frequenza dell'elemento 1 è $h$ mentre quella dell'elemento $\mathrm{O}$ è $1-h$, si ha

$$
k=\frac{1}{h}
$$

In prescnza di disturbi, se $\grave{e}$ stato trasmesso un segnale elemenlare 1 , si avrà un errore (in energia) dato da

$$
\varepsilon_{1}=\left|R \int_{i}^{t+\tau} e_{\mathrm{s}}^{2} d t-k W_{\mathrm{m}}\right|
$$

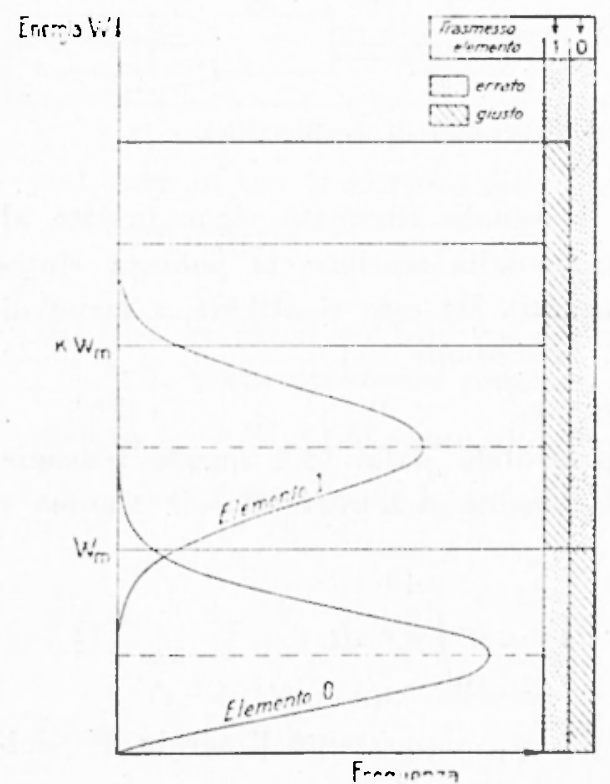

Fig. 4 - Possibile distribuzione dei segnali elementari ricevuti.
Se invece il segnale elementare trasmesso è $\mathrm{O}$, l'errore (in energia) sarà dato da

$$
\varepsilon_{0}=R \int_{t}^{t+\tau} e_{\mathrm{s}}^{2} d t-0
$$

La distribuzione statistica dell'energia all'uscita per ciascun elemento potrà essere del tipo rappresentato in fig. 4.

Definiamo ora segnale errato quello per cui l'errore in energia è maggiore li $\frac{1}{2} k \Pi_{n}^{r}$, e segnale giusto quello per cui l'errore in energia è minore di questa entita. (Il caso in cui l'errore in energia è uguale a 
$\frac{1}{2} k W_{\text {m }}$ ha probabilita trascurabile). Questa definizione ha, su altre possibili, la proprietà della simmetria e quella di considerare che qualsiasi elemento riceruto vengra decodificato, senza ambiguitì.

La intellegibilità misurata sarà ug̣uale al rapporto tra numero dei segnali giusti e numero dei segnali trasmessi, quindi uguale al complemento a 1 del rapporto tra numero dei segnali errati e numero dei sernali trasmessi.

4. Programmazione del calcolo. - La fig. 5 presenta lo stenogramma del registratore di intellegililita.

4. 1. Il segnale proveniente dal ricevitore attraversa un attenuatore manuale con eui se ne regola l'intensita in modo da rimanere

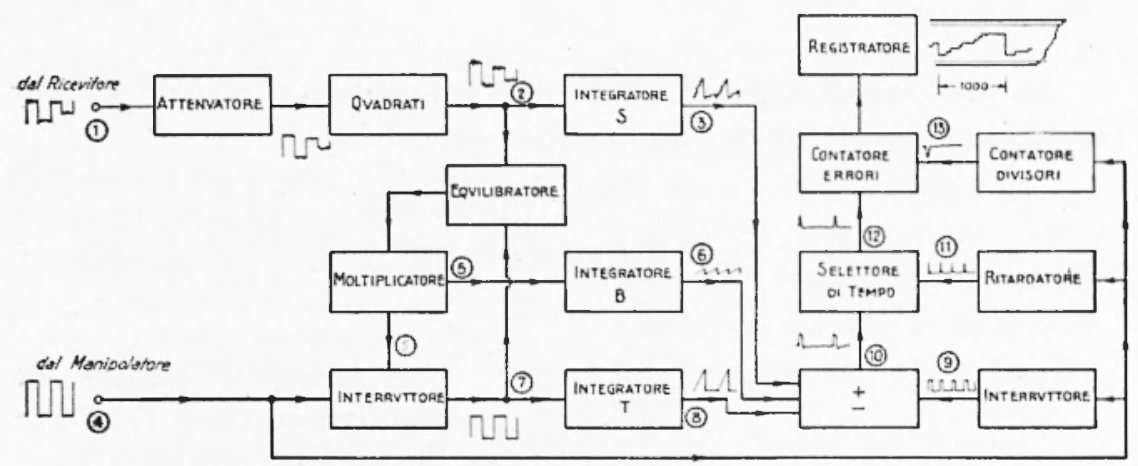

Fig. 5 - Stenogramma del registratore di intelligibilita.

nel campo di autoregolazione. Il segnale attenuato viene inviato al circuito dei quadrati, per oltenere dalla tensione la potenza (integrando della [1], a meno di costanti). Da esso si ottiene, a meno di una costante di moltiplicazione, la tensione

$$
p_{\mathrm{S}}=e,
$$

Per ottenere l'energia rappresentata dalla [1], questa tensione alimenta l'integratore $S$, che al termine dell'intervallo di tempo $\tau$ fornisce una d.d.p.

$$
q_{\mathrm{s}}=b \int_{t}^{t+1-\tau} p_{\mathrm{s}} \cdot d t=a R \int_{i}^{t+\tau} e_{\mathrm{s}}^{2} d t
$$

in cui $a$ e $b$ sono costanti. La d.d.p. $q_{s}$ rappresenta l'energia $W$ della $[1]$, a meno della costante $\frac{1}{a}$. 
4. 2. Con la [7] si è ottenuto il minuendo della [4] o [5]. Per ottenere il sottraendo il manipolatore comanda un interruttore dal quale si ottiene una tensione manipolata, la cui ampiezza è regolata come segue.

La tensione manipolata $P_{\mathrm{T}}$, e la tensione uscente dal circuito dei quadrati $p_{\mathrm{s}}$, vengono confrontate nell'equilibratore. Questo ì un amplificatore differenziale in continua, che corregge l'ampiezza della tensione da manipolare, per rendere uguali la media della tensione uscente dal circuito dei quadrati $p_{\mathrm{s}}$ e la media della tensione manipolata $p_{\mathrm{T}}$. Ciascuna di queste due tensioni medie rappresenta allora, a meno di una stessa costante di moltiplicazione, la energia media $W_{\mathrm{m}}$.

La [2] non è direttamente applicabile. Un valore approssimato della media viene ottenuto filtrando la tensione $p_{s}$ attraverso ad un circuito $R C$ con costante di tempo scelta per compromesso tra l'errore di campionatura e la possibilita di seguire le flutuazioni dei disturbi.

L'integratore $T$ ha la funzione di fornire la tensione rappresentativa dell'energia che si riceverebbe in assenza di disturbi. Perciò la tensione manipolata $p_{r}$ viene integrata nell'integratore $T$ ed alla fine dell'intervallo di tempo $\tau$ si ottiene una d.d.p.

$$
\tau_{\mathrm{T}}=b \int_{i}^{t+\tau} p_{\mathrm{r}} d t=a k W_{\mathrm{m}}
$$

nel caso in cui il circuito sia chiuso (segnale 1).

Sarà invece

$$
p_{\mathrm{I}}=0
$$

nel caso in cui il circuito sia aperto (segnale $O$ ).

4. 3. 亡 energia $\frac{1}{2} k \Pi_{m}$. Ciò viene ottenuto nell'integratore $B$. che viene alimentato dall'equilibratore e fornisce una d.d.p.

$$
q_{\mathrm{B}}=a \frac{1}{2} k W_{\mathrm{m}}
$$

4. 4. Le tre d.d.p. suddette rengono inviate in un circuito che fornisce le differenze

$$
\begin{aligned}
& د=q_{\mathrm{s}}-q_{\mathrm{s}}-q_{\mathrm{s}} \\
& \Delta=q_{\mathrm{s}}-q_{\mathrm{s}}-q_{\mathrm{B}}
\end{aligned}
$$


Se una delle due differenze suindicate è maggiore di zero allora si deve contare un errore $\left(^{*}\right)$. Per fare ciò, il circuito delle differenze viene attivato soltanto alla fine dell'intervallo di integrazione, mediante l'intervento di un interruttore su ciascuna delle d.d.p. integrali, per un tempo di circa 500 / us (tempo di calcolo). Alla metá di questo tempo di calcolo un circuito selettore di tempo fa passare un impulso preformato se, e soltanto se, una delle differenze [11] o [12] ̀̀ maggiore di zero, cioè se deve essere contato un errore. In questo caso l'impulso fa avanzare di una unità il contatore di errori.

4. 5. Il contatore dei divisori viene invece fatto avanzare di una unità per ognni elemento trasmesso. Quando il contatore dei divisori ha contato 1000 elementi, esso ritorna a zero e riporta a zero il contatore errori. In questo istante il numero letto nel contatore errori ̀̀

(*) Se lelemento trasmesso è 1 , posto

$$
\frac{\Delta}{a}=\left|\varepsilon_{1}\right|-\frac{1}{2} k W_{\mathrm{m}}=\left|R \int_{i}^{t+\tau} e_{i} d t-k\left\|_{\mathrm{m}} \mid \frac{1}{2} k\right\|_{\mathrm{m}}>0\right.
$$

si ha anche

$$
\Delta=\left|a R \int_{t}^{t+\tau} e_{\mathrm{a}}^{2} d t-a k \|_{\mathrm{m}}\right|--a \frac{1}{2} k \Pi_{\mathrm{n}}=\left|\eta_{\mathrm{S}}-q_{\mathrm{T}}\right|-q_{\mathrm{B}}>0
$$

che si scinde in

oppure

$$
\Delta_{\mathrm{l}}=q_{\mathrm{S}}-q_{\mathrm{T}}-q_{\mathrm{B}}>0
$$

a seconda che sia

$$
\Delta_{1}=g_{T}-q_{\mathrm{S}}-q_{\mathrm{B}}>0
$$

oppure

$$
q_{\mathrm{S}}-q_{\mathrm{T}}>0
$$

$$
q_{\mathrm{S}}-q_{\mathrm{T}}<0
$$

Se invece l'elemento trasmesso $\dot{e} \cap$, posto

$$
\frac{\Delta}{a}=\left|\varepsilon_{0}\right|-\frac{1}{2} k \Pi_{\mathrm{m}}=R \int_{t}^{i+\mathrm{r}} e_{\mathrm{e}}^{2} d t-\frac{1}{2} k H_{\mathrm{m}}>0
$$

si ha anche

$$
\Delta=a R \int_{t}^{t+\tau} e_{\mathrm{i}}^{\tau} d t-0-a \frac{1}{2} k r_{\mathrm{m}}=q_{\mathrm{S}}-q_{\mathrm{T}}-q_{\mathrm{B}}>0
$$

che è ancora la [11]. 
uguale al rapporto tra il numero dei segnali errati ed il numero degli elementi trasmessi, moltiplicato per 1000.

4. 6. Mediante una rete di resistenze ed una valvola amplificatrice, un registratore a penna segue il contatore di errori. L'elongazione della penna, in ciascuna escursione, indica perciò il rapporto tra il numero dei segnali errati ed il numero degli elementi trasmessi, ciò̀ il complemento a 1 dell'intellegibilità.

Con l'avanzamento della carta si ottiene un diagramma in cui l'intellegibilità compare in ordinata contro il tempo in ascissa (fig. 11).

4. 7. I circuiti permettono il funzionamento a velocità da 10 a 50 haud, e possono utilizzare tanto l'uscita di un ricevitore, a frequenze acustiche fino a $1000 \mathrm{~Hz}$, quanto direttamente la corrente di ingresso di dispositivi meccanici (relè, ondulatore). Il campo di funzionamento copre la maggior parte dei casi di comunicazione singola, ma con qualche modifica potrebbe venire esteso anche a quello di canali multipli.

5. Circuito dei quadrati, equilibratore, integratori. - Il segnale proveniente dal ricevitore, mediante i tubi $V 3$ e $V 4$, comanda in controfase i tubi $V 5$ e $V 6$ (fig. 6). Il valore medio delle griglie di questi luhi viene regolato a $12,5 \mathrm{~V}$. Sulla resistenza comune di carico $R 23$ si otliene una caduta di tensione proporzionale al quadrato della tensione all'ingresso (a meno di una costante additiva). V 8 inverte la fase. $V 9$, insieme al condensatore $C 6$, elimina la costante additiva. Nel punto 2 si ha perciò una tensione proporzionale alla potenza del segnale ottenuto dal ricevitore.

Il condensatore $C 7$ attraverso la resistenza $R 30$ acquista una carica proporzionale all'integrale di questa tensione, cioè all'energia del segnale.

Alla fine dell'intervallo di integrazione il tubo a gas $V 10$ scarica il condensatore. Perché la scarica avvenga sicuramente alle tensioni di pochi volt a cui il condensatore si trova caricato, il catodo di $V 10$ è connesso ad una tensione di $-90 \mathrm{~V}$. A fine scarica $C 7$ è negativo, e viene portato a zero dal diodo $V 11$.

$V 1$ alimenta un altoparlante spia; $V 2$ comanda i tiratron. $\bar{V} 2$ riceve, alla fine dell'intervallo di integrazione, un rettangolo di tensione negativo di $500 \mu$ s. Lo scatto di tensione della fine di questo rettangolo, attraverso al condensatore $C 8$, comanda la prima griglia del tetrodo a gas.

Nel punto 5 si ha la tensione normalizzata di ingresso degli in- 


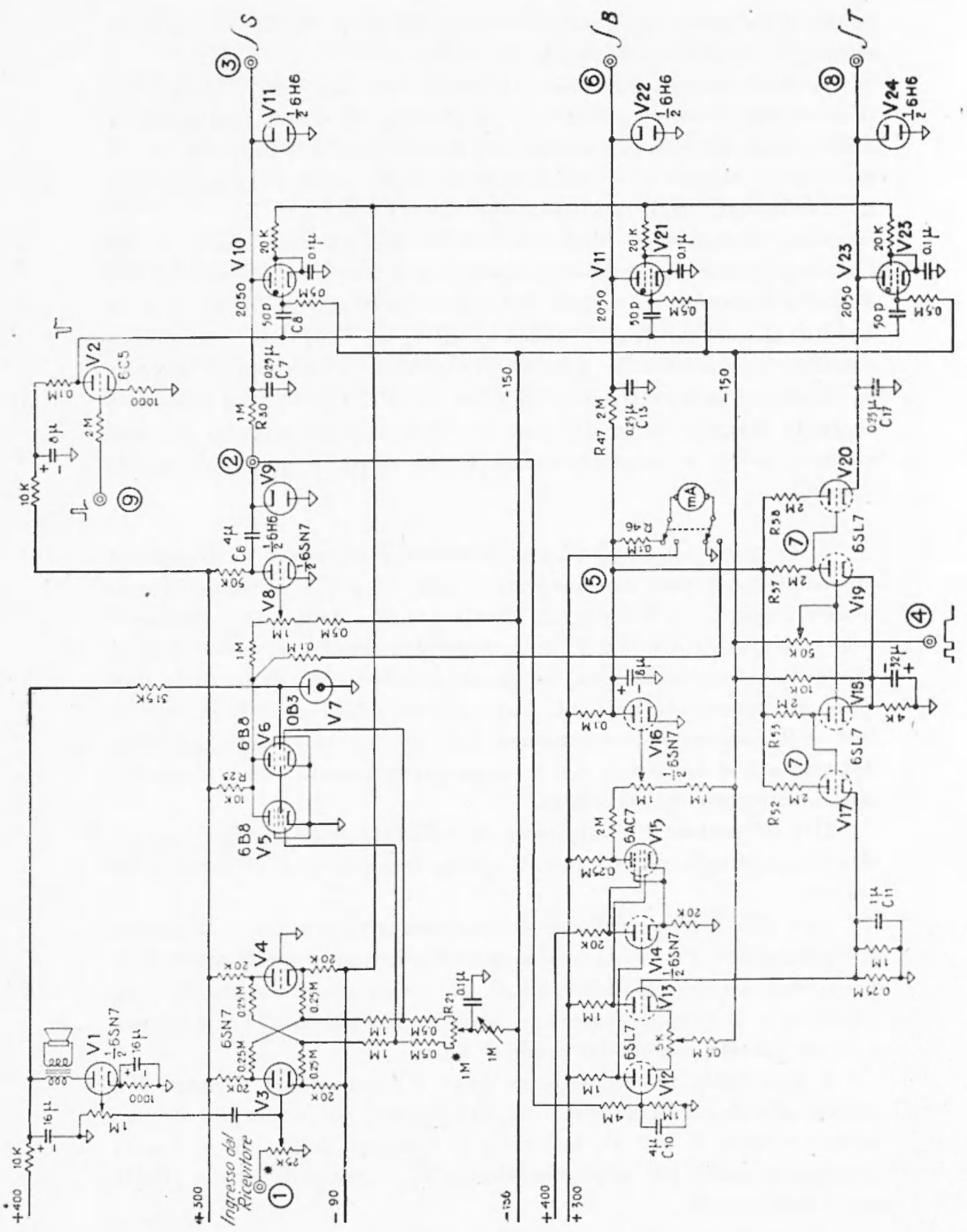


tegratori $B$ e $T$. L'inlegrazione $B$ aviene nel circuito $R+7 C 13$, con $V 21$ e $V 22$ che funzionano come $V 10$ e $V 11$.

I tubi $V 19$ e $V 20$ manipolano la tensione normalizzata. Nelle resistenze $R 57$ ed $R 58$ in parallelo tra loro ed in serie con la resistenza interna di $V 20$, e nel condensatore $C 17$ avviene l'integrazione $T$. V 23 e $V^{\prime} 24$ sono analogrhe a $V 10$ e $V 11$.

Ai capi di $C 11$ si otliene una frazione della tensione che alimenta $C$ 17. Su $C 11$ si ha quindi la media del valore all'ingresso dell'integratore $T$. D'altra parte, ai capi di $C$ 10 si ha la stessa frazione della media della tensione all'ingresso dell'integratore $S$.

$V^{*} 12, V^{*} 13, V 1 ., V^{*} 15, \quad V 16$ costituiscono un amplificatore in continua che, attraverso al circuito di placca di $V 17$ (comandata da $V$ 18) funziona in controreazione. Quando si verifica una differenza tra le due medie tale amplificatore fa variare la tensione in 5 , che cosi viene normalizzata.

Il voltmetro, costituito da $R 46$ e dal milliamperometro in serie, consente di accertarsi che l'equililoratore funzioni entro il suo campo di regolazione.

Lo stesso strumento, mediante il commutatore, permette di regolare l'esatto equililirio fra le tensioni di comando dei tuli $I 5$ e V 6, nonche, mediante altre commutazioni non indicate in figura, di verificare le tensioni di alimentazione.

6. Formazione del segnale di errore. - Le uscite dei tre integratori sono connesse a tuli ad uscita catodica $V 31, V 32, V 33$, che scparano orli integratori dal circuito di somma e differenza che segue ifig. i. I 3.t ha lo scopo di produre una tensione che compensa la parte costante di quella data dal tubo ad uscita catodica $V 33$.

V36. V 37 . V 38 e 139 costituiscono degli interuttori che, comandati in parallelo da $V 35$, applicano le tensioni all'ingresso del circuito di somma e differenza. Tale circuito è costituito dalle resistenze $R$ 116....R 123, e dai tul,i $V 40, V 41, V 42, V 43$, ed effettua le operazioni indicate dalle espressioni [11] e [12]. Attraverso ai condensatori $C 105$ e $C 106$ i risultati delle operazioni rengono applicati ai tuhi amplificatori $V+4$ e $V \mathbf{t 5 .}$

$\checkmark 46$ e $V 47$ effettuano l'operazione logica “oppure ». Infatti basta che uno di essi ricera un rettangolo di tensione positivo sulla griglia affinché si generi un rettangolo di tensione negativo sulla comune resistenza di carico $R$ 150. Questo rettangolo negativo ha la 


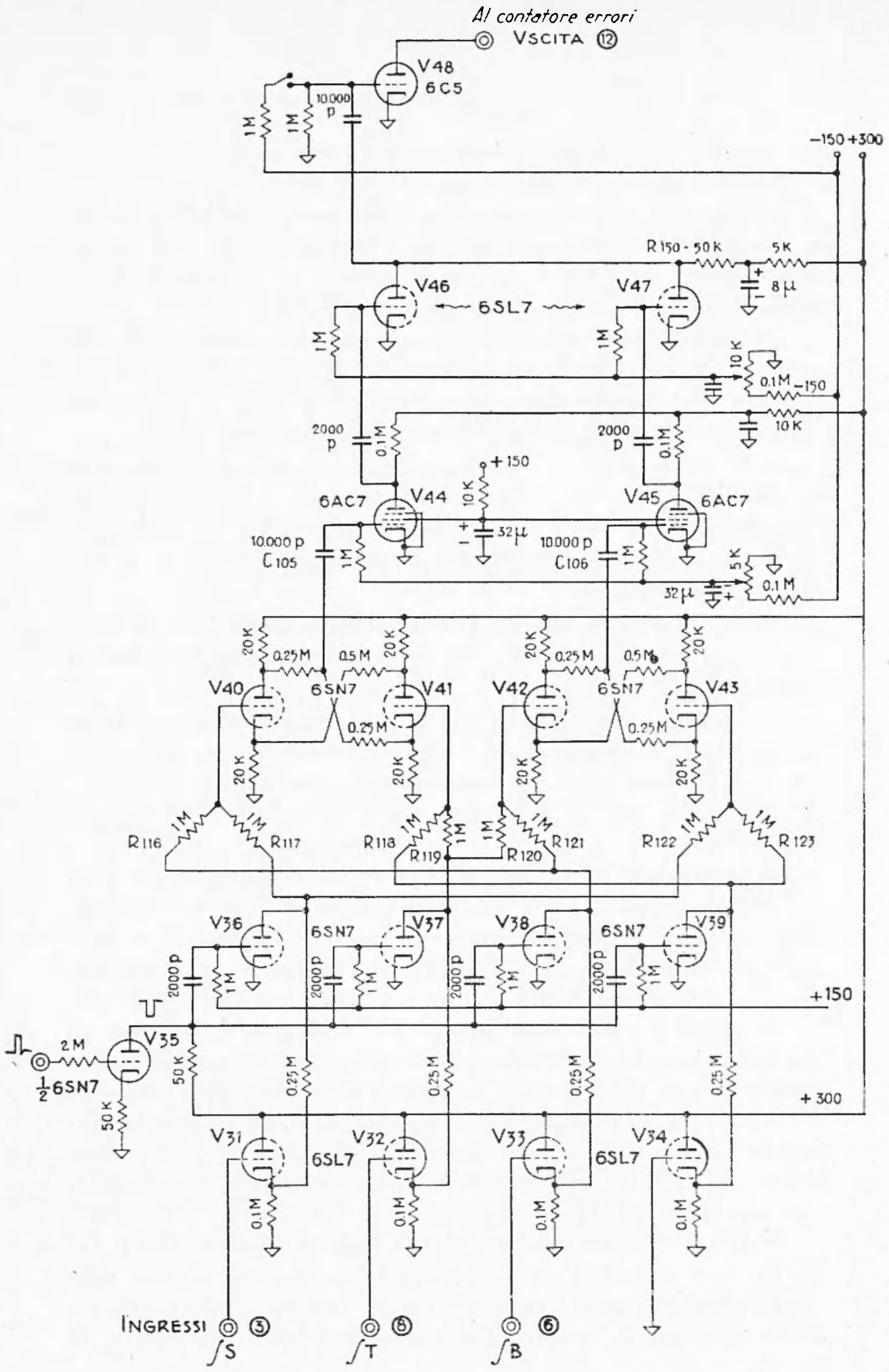

Fig. 7 - Comparatori di segnali. 
durata di $500 \mu s$, ma in conse. guenza degrli accoppiamenti $R C$ tra i tubi precerlenti esso è segruito da una corla. Non può quindi venire utilizzato direttamente per comandare il contatore di errori. Perciò V 51 (fig. 8) applica a C. 115 un retlangolo negativo della durata di $200 \mu \mathrm{s}$. Lo scatto finale di questo rettangolo attraverso a $C$ 115, da luogo ad un impulso positivo centrato rispetto al rellangolo di 500 !ıs. I rettangoli di 200 e di 500 us sono generati con inizio approssimativamente coincidente. L'impulso positivo uscente da $C 115$ viene cortocircuitato da $V \cdot 18$ a meno che sulla rriplia di questo tubo sia applicato il rettangolo negativo di $500)$ us renerato da $V 46$ oppure da $V 47$. Soltanto in questo caso il contatore di errori procede di una uniti. La griglia di $V 48$ può essere resa negativa anche manualmente, allo scopo di verificare il funzionamento dei circuiti successivi.

\section{Contatori. - Il contatore} di errori (fig. 8) è una scala a 32 di tipo consueto; l'ingresso ad essa aviene dopo limitazione, attraverso al doppio diodo V52 V53, mentre l'acoppiamento tra gli stadi successivi è per $R C$. $I^{*} 55$ normalmente conduce, e chinde il circuito di griglia del tubo zero dei diversi stadii. Quando alla griglia di $r 55$ viene applicato

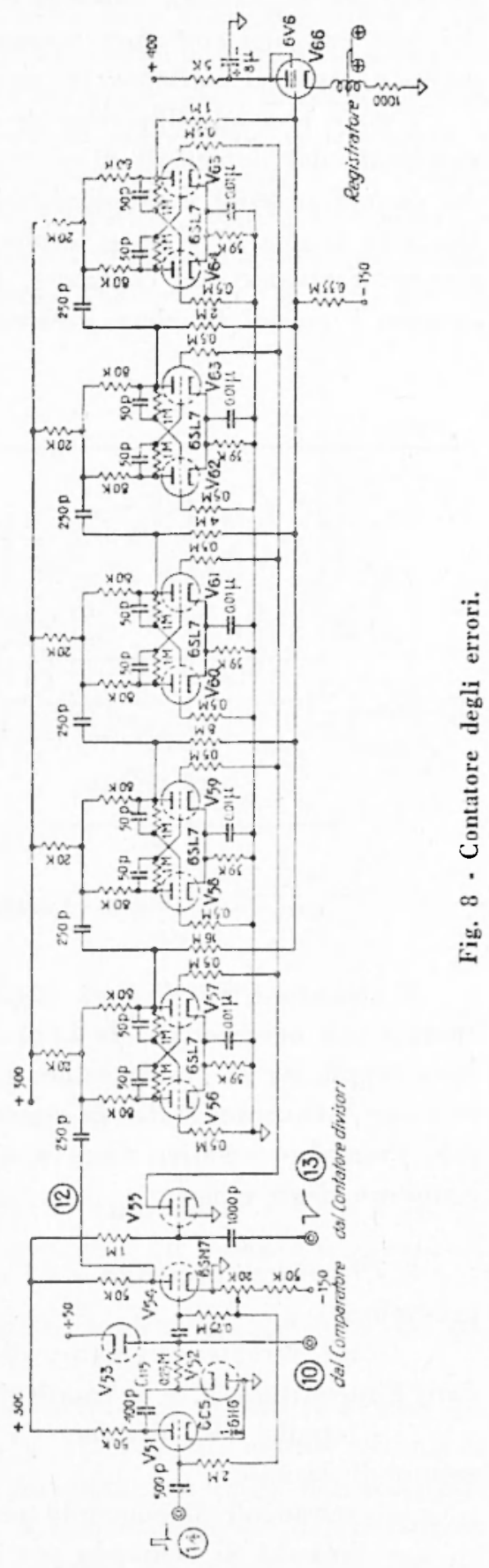


un impulso negativo proveniente dal contatore dei quozienti, le griglie dei tubi zero del contatore possono diventare positive, e si ha la rimessa a zero del contatore di errori.

V 66 è una amplificatrice di corrente, comandata da una media ponderata dei potenziali di placea dei tubi zero della scala. Il peso dei diversi addendi è proporzionale alle successive potenze di 2, così che alla griglia di $V 66$ è applicato un potenziale proporzionale al numero registrato dal contatore. L'elongazione della penna del registratore $\grave{e}$ quindi funzione crescente di questo numero.

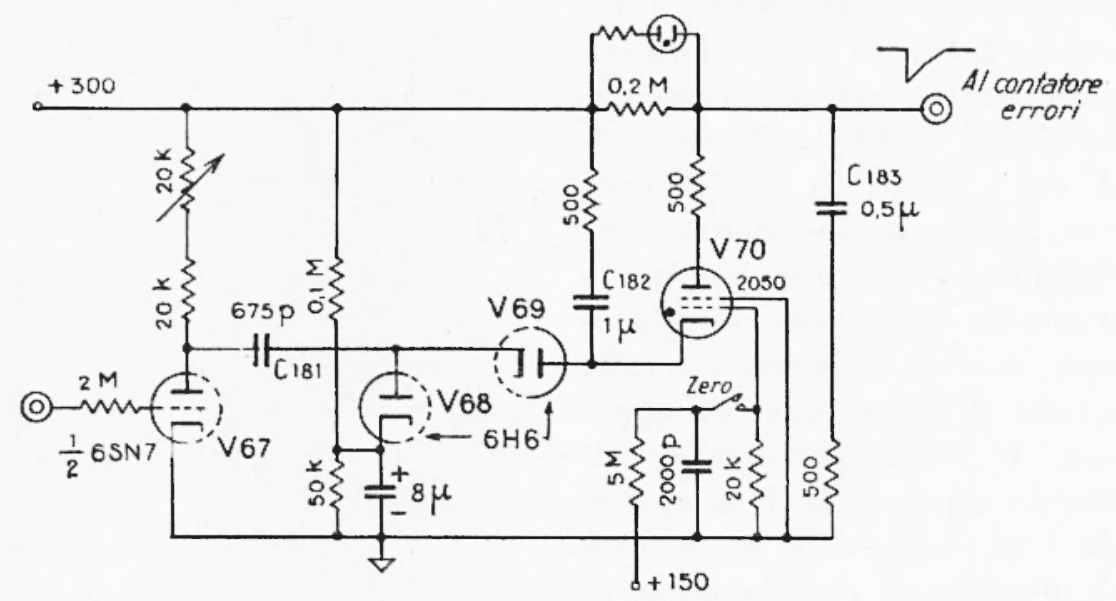

Fig. 9 - Contatore dei divisori.

Il contatore dei divisori (fig. 9) è del tipo ad accumularione di energia. Ad ogni rettangolo applicato alla griglia di $V 67$, una carica viene applicata al condensatore $C$ 182. Quando $C 182$ ragionge una tensione sufficiente, si ha la scarica attraverso a $V 70$, e viene generato l'impulso negativo che va a comandare la rimessa a zero del contatore degli errori.

8. Circuiti di comando. - I circuiti di comando (fig. 10) devono provvedere:

- un segnale campione, di ampiezza conveniente per comandare l'integratore $T$, e a fronti ripidi;

- impulsi che dividano il tempo in intervalli uguali allelemento di segnale;

- rettangoli di comando per gli intervalli di calcolo:

- impulsi di comando per il contatore di errori. 
Con i commutatori nella posizione "Est " il segnale viene introdotto dal morsetto 4 sotto forma di una tensione manipolata oppure di chiusura ed aperture di circuito verso massa. Alle placche del multivibratore histabile $V 71 \quad V 72$ si ottiene un segnale di ampiezza e forma normalizzata. Questo segnale sincronizza, tramite $V 74$, il multiviluratore astabile $V 75 V 76$, la cui frequenza propria è regolata variando $R 201$ e $R 202$ - sulla velocità di manipolazione. Si ottiene cosi un fronte ripido in corrispondenza alle divisioni in elementi del seqnale.

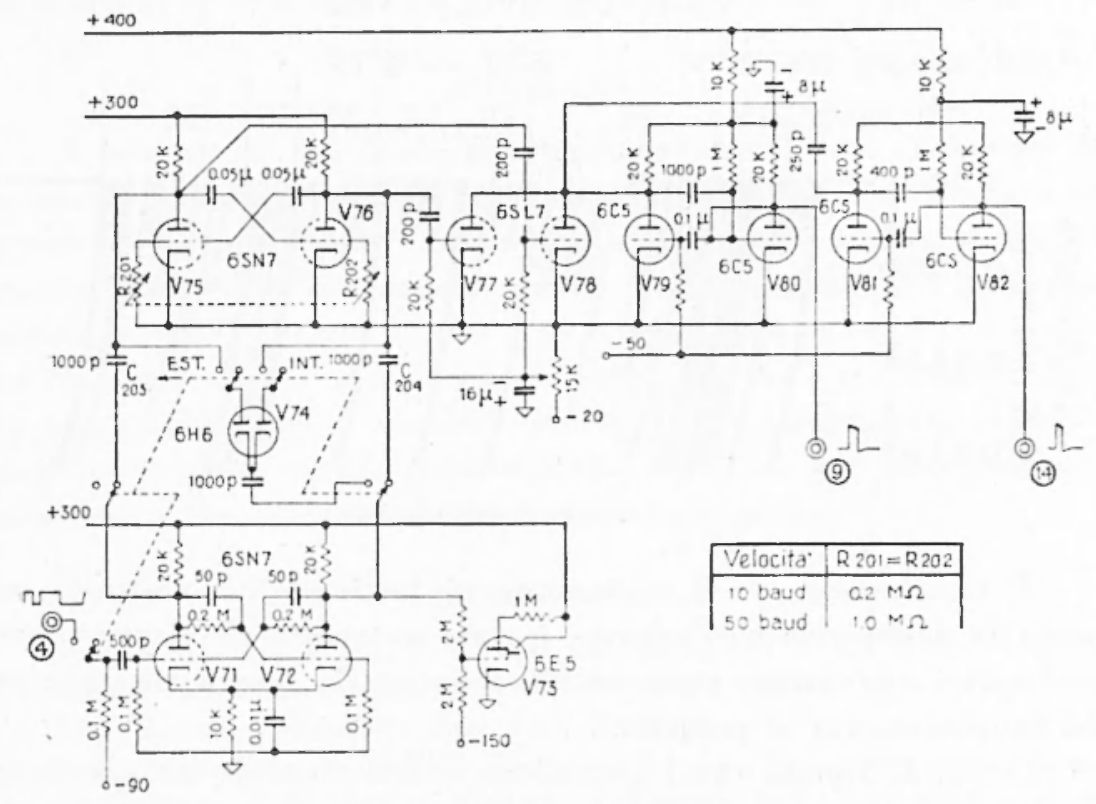

Fig. 10 - Circuiti di comando.

1 fronti ripidi, derivati dai circuiti di griglia di $V 77$ e $V 78$ comantano le placche di $V 79$ e $V 81$ mediante un impulso negativo, ad ogni fine elemento.

$V 79$ e $V 80$ costituiscono un multiviluratore monostalile che genera un rellangolo 9 di cirea 500 us.

I 81 e $V 82$ costituiscono un multiviloratore monostabile che genera un rettangolo $\mathrm{It}$ di cirea $200 \mathrm{nt}$. L'inizio di questo rettangolo ì leggermente ritardato rispetto al precedente, a causa dell'accoppiamento capacitivo utilizzato. Quindi il lato discendente del rettangolo viene a trovarsi approssimativamente a metà del reltangolo di $500 \mu$, 
e per derivazione può generare l'impulso 11 (fig. 5) di conıundo del contatore errori, attraverso il selettore di tempo.

Il comparatore può anche essere disposto per funzionare senza comando esterno In questo caso, con il commutatore sulla posizione "Int ", è il multivibratore astabile $V 75 V 76$ che, tramite i condensatori $C 203 C 204$, comanda il multivibratore bistabile $V 71 \quad V 72$. Il segnale generato è allora un'onda quadrata. Il segnale stesso deve venire prelevato (dal multivibratore bistabile) per manipolare il generatore di segnali campione.

\section{0 baud $-2,5 \mathrm{MHz}$}

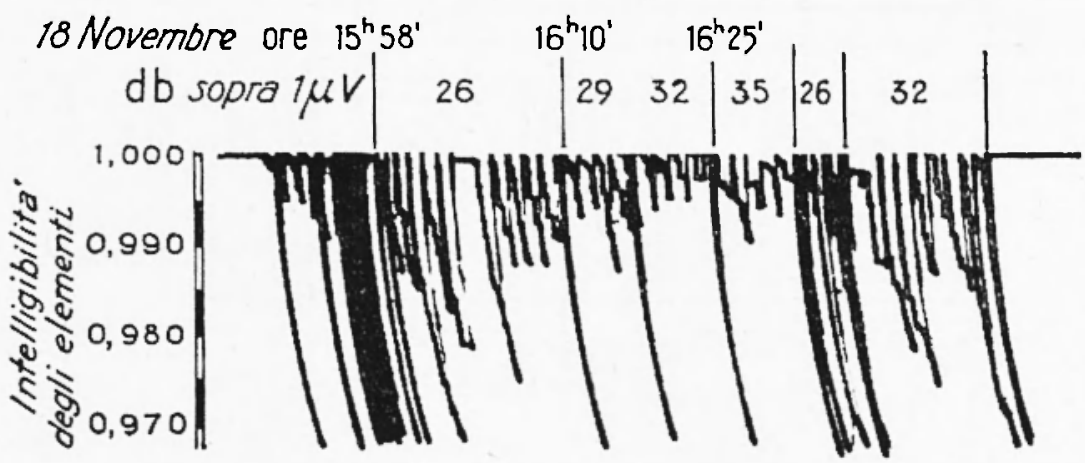

Fig. Il - Esempio di registrazione.

9. Conclusione. - Il registratore di intelligibilità, costruito secondo la descrizione che precede, è stato sottoposto ad alcune prove preliminari che hanno permesso di accertare la buona rispondenza del funzionamento al progetto.

La fig. 11 riporta una registrazione ottenuta usando un ricevitore $B C-312-N$ ed un generatore di segnali campione Marconi-Ecko mod. T.F. $144 F$; antenna di circa $6 \mathrm{~m}$ di altezza.

I dati riportati nella figura non sono rappresentativi della intensità dei disturbi nella località di ricezione (Roma), mancando indicazioni precise sulla larghezza di banda del ricevitore e sulle caratteristiche dell'antenna (del resto poco adatta). È però possibile osservare nel diagramma la sensibile variazione di intelligibilità che si ha con variazioni di intensità dei segnali di 3 decibel.

Per effettuare registrazioni sistematiche occorrerà un impianto analogo a quello delle stazioni Thomas, accennato nell'introduzione e descritto da Thomas e Cottony ( ${ }^{1}$ ).

Roma - Istituto Nazionale di Geofisica - Novembre 1952. 


\section{RIASSUNTO}

$\grave{E}$ stato costruito, presso l'Istituto Nazionale di Geofisica, un comparatore di segnali che permette di registrare la percentuale di errori nella ricezione di un segnale radiotelegrafico disturbato. L'apparecchio, costituito da una calcolatrice elettronica analogico-numerica, tende ad eliminare $i$ fattori soggettivi che normalmente si hanno in que. sto tipo di misure, ed è destinato principalmente alla misura dei radio disturbi atmosferici con metodo sintetico per confronto con un segnale campione. Il dispositivo si presta anche a misure comparative su ricevitori ed antenne, ed a misurare l'effetto delle interferenze.

\section{$S U M M A R Y$}

A comparator has been constructed, at the Istituto Nazionale di Geofisica, uhich records the percentage of errors in the reception of a radio telegraph signal. The purpose of this upparatus, consisting of an electronic digital-analogue computer, is to eliminate the subjective factors normally present in this type of measurement, and is destined for the measurement of atmospheric radio noise by a stnthetic method by comparison with a standard signal. This apparatus can also be used to compare signals from different receivers and antennas and to measure the effect of interference.

\section{BIBLIOGRAFIA}

(t) H. A. Thonas and H. V. Cottosy, Interim. Report on the Measurement of Atmospheric Noise Level. N.B.S. Report CRPL - 5.l (genn. 1947).

(2) N. Wiener, Cybernetics. New York (1948).

(3) G. E. Valtey, Jr. and H. Wallana, Vacuum Tube Amplifiers. Massachussetts Institute of Technology, Radiation Laboratory Series, vol. 18, New York (19.49).

(t) B. Chance, V. Hughes, E. F. MacNicol, D. Sayre and F. C. Willanis, Faveforms. Massachussetts Institute of Technology, Radiation Laboratory Series, vol. 19, New York (1949).

(5) A. Bolle, S. Sillfeni e C. A. Tinfrio, Registrazioni ionosferiche. "Annali di Geofisica ", vol. II, pag. 37î-387 (luglio 1949).

(6) H. O. Peterson, Discussion relative to radio noise. "U.R.S.I., Proc. of the General Assembly held in Zurich from September llth to 22nd, 1950" vol. VIII, part. II, Bruxelles, 1950, pag. 289.

(7) S. Sillent, Misura dei radio disturbi almosferici. « Rendiconti dell'A.E.I. ", Bologna, (17-2t settembre 1950).

(8) P. M. Woodwand and I. L. Davies, Information Theory and Inverse Probability in Telecommunication. "Proc. I.E.E. ", vol. 99, part. III, pag. 37.44 (marzo 1952). 